\title{
„Wie die virtuelle Welt uns zu narzisstischen Multitaskern gemacht hat."
}

\section{Ulrich Heitzlhofer zu Erfahrungen aus der Personalentwicklungsarbeit während der COVID-19-Krise}

\author{
Ulrich Heitzlhofer ${ }^{1,2} \cdot$ Karin Lackner ${ }^{3}$ \\ Online publiziert: 10. Februar 2022 \\ (c) The Author(s), under exclusive licence to Springer Fachmedien Wiesbaden GmbH, ein Teil von Springer Nature 2022
}

\section{Zusammenfassung}

Mag. Ulrich Heitzlhofer ist Leiter der Personalentwicklung mit den Schwerpunkten auf Weiterbildung und Leistungsevaluierung im Rideshare Unternehmen Lyft. Zu seinen Tätigkeiten gehören die (Re)Konstruktion des gesamten Leistungsevaluierungsprozesses, die Entwicklung eines Weiterbildungsportfolios und die strategische Ausrichtung einer firmenweiten Personalentwicklung.

Die COVID-19 bedingten Umstellungen des gesamten Personalentwicklungs- und Weiterbildungsbereiches kennzeichneten die Herausforderung der letzten 1 1 12 Jahre und führten zu überraschenden Innovationen des gesamten Bereiches.

Schlüsselwörter Personalentwicklung · Veränderungsmanagement · COVID-19-Krise · Digitalisierung · Home-Office Arbeit

\section{"How the digital world turned us into narcissistic multitaskers."}

Ulrich Heitzlhofer on experiences from personnel development work during the COVID-19 crisis

\begin{abstract}
Uli Heitzlhofer is currently heading Lyft's People Development function where he oversees the strategic alignment of learning \& development initiatives as well as Talent Management processes to company wide objectives.

Adjustments and re-evaluation of People Development/Talent Management practices due to COVID-19 have been a major challenge for the past $1 \frac{1}{2} 2$ years, yet, these shifts also revealed surprising upsides.
\end{abstract}

Keywords Human resources · Change management · COVID-19 crisis · Digital works · Home office

Das Interview mit Ulrich Heitzlhofer führte Karin Lackner.

Ulrich Heitzlhofer

heitzlhofer@gmail.com

Karin Lackner

ifo.lackner@gmail.com

1 Personalentwicklung, Rideshare Unternehmen Lyft, 185 Berry Street, San Francisco, CA, 94107, USA

2 Vienna University of Economics and Business, Welthandelsplatz 1, 1020 Wien, Österreich

3 Institut für Organisationsdynamik, Arsenal 16/75, 1030 Wien, Österreich
Was waren die größten Veränderungen in Ihrem beruflichen und privaten Alltag, die durch die COVID-19 Krise und den damit verbundenen Lockdowns hervorgerufen wurden? Von zu Hause aus zu arbeiten, sich ein virtuelles Office einzurichten, damit dieses arbeitstechnisch funktioniert, war wohl die erste große Herausforderung. Beruflich, auf die Personalentwicklung und die Arbeitsprozesse in diesem Bereich bezogen, mussten wir alles, was wir anbieten, an einen virtuellen Kontext anpassen. Alle Veranstaltungen und Workshops, die in Präsenz durchgeführt wurden, mussten Schlag auf Schlag auf digital umgestellt werden. 
Die Herausforderungen dabei waren mehrfach. Erstens die Technologie: bis zu diesem Zeitpunkt hatte wir zwar bereits mit verschiedensten Technologien gearbeitet, aber deren Einsatz war eher die Ausnahme als die Norm. Neue technologische Lösungen mussten an die jetzt bestehenden Anforderungen angepasst werden. Zum Beispiel stellte sich heraus, dass Google Meet nicht das optimale System für Online-Weiterbildungsprogramme war. Die Software ist toll für die Kommunikation, nicht jedoch um verschiedene Weiterbildungs-Designs online einzubinden. Es war ,unangenehm spannend“ diese Systeme an ihr Limit zu bringen und unsere Designs an diese Limitierungen anzupassen.

Der erste Schritt dieser Herausforderung war es, unsere gesamte Bandbreite an Themen auf ihre virtuellen Anpassungsmöglichkeiten hin zu durchleuchten. Wir haben einen Monat lang alle Programme gestoppt und uns nur damit beschäftigt, alles für den virtuellen Raum abzuändern und alles, was virtuell möglich war, sofort umzusetzen.

Welche Überraschungsmomente gab es im Zuge dieses Prozesses? Ein zweitägiges Workshop-Programm für neue Manager war im virtuellen Bereich nicht machbar. Wir konnten die Teilnehmenden nicht zwei Tage lang für eine Online-Präsenz verpflichten. Das war hart, denn dieses Programm war eines unserer besten. Wir haben die Veranstaltung in 2-Stunden-Einheiten pro Woche über eine Periode von vier Wochen durchgeführt.

Das hat überraschend gut funktioniert. Erstens sind die Teilnehmenden nicht nur gekommen, sie sind auch geblieben und waren sehr engagiert. Am überraschendsten war, wie erfolgreich die asynchrone Arbeit, die zwischen den jeweiligen Workshops von Woche zu Woche stattfand, ablief, wo Teilnehmende an den Aufgaben arbeiteten, die ihnen in den Workshops mitgegeben wurden. Die Aufgaben haben wir in den Kleingruppen erstellt, die sich selbstorganisierend trafen und sich mit den jeweiligen Themenbereichen, die wir in den zwei Stunden angesprochen hatten, auseinandersetzten. Das hat, im Nachhinein betrachtet, der Kurve des Vergessens ${ }^{1}$ entgegengesteuert. Beim jeweils nächsten Treffen wurde kurz wiederholt was man beim letzten Mal besprochen hatte und darauf aufgebaut.

Ein ähnliches Programm haben wir für die Direktor:innen organisiert. Das ursprüngliche Programm eines 1-Tages-Workshops haben wir seit COVID-19 digital ebenfalls auf zwei Stunden pro Woche über einen Zeitraum von vier Wochen aufgeteilt, und gleich wie bei den Managern Kleingruppen gebildet. Die Einladungen zu diesen Treffen kamen immer von den jeweiligen Top-Führungskräften, nicht von uns aus der Personalabteilung. Damit ist das Engagement, sich immer wieder zu treffen, gestiegen.

\footnotetext{
${ }^{1}$ https://en.wikipedia.org/wiki/Forgetting_curve.
}

Sind solche Innovationen im Personalentwicklungs- und Weiterbildungsbereich wie beispielsweise solche begleiteten Selbststeuerungsformate in der COVID-19 Zeit entstanden? Diese Kleingruppen, - wir haben sie „Peer Group Coaching" genannt - hatten wir schon vor COVID-19. Aber es hat nie so richtig gut funktioniert, weil wir es entweder immer selbststeuernd belassen hatten oder wir waren dort selbst die Moderator:innen. Wir hatten nie daran gedacht, dass wir eigentlich jemand aus den BusinessBereichen hinzuziehen könnten, der mit uns gemeinsam das Programm gestaltet - das ist uns wirklich erst aufgrund der nun bestandenen sozialen Distanz eingefallen. In den Kleingruppen, die sich von Woche zu Woche getroffen hatten, gab es jeweils Repräsentant:innen, die Dokumentationen erstellt hatten. Unsere Unterstützung bestand darin, die Administration des Gesamtprozesses $\mathrm{zu}$ organisieren und die Co-Facilitators vor jedem Meeting zu briefen. Zudem boten wir diesen Personen Coaching für die Moderation der Peer Group Coaching Gruppen an. Dieses System haben wir bei Direktor:innen gemacht, aber auch bei Vice Presidents, und das ging genau gleich gut.

Weiters haben wir jene Knotenpunkte in der Organisation identifiziert, wo Personen schon von vornherein gut verknüpft waren. Wer sind die Personen, von denen sich alle anderen wie durch einen Magnet angezogen fühlen. Das betrifft bei Tech Unternehmen vor allem Mitarbeitende aus dem Produktmanagementbereich. Sie spielen eine zentrale Rolle, denn mit ihnen müssen sich alle anderen Bereiche auseinandersetzen. Diese Personen schlüpfen dann oft in die Moderator:innen-Rolle der Peer Coaching Gruppen.

Die große Überraschung war, dass das so gut gelungen ist, und auch dass sich die Erkenntnis bestätigt hat, dass, wenn man Mitarbeitenden das „Empowerment“ gibt, sie wesentlich engagierter sind. Wichtig war es, das Business in die Gestaltung mit einzubeziehen und den aktiven Personen eine Anreiz zu geben (z. B. Coaching).

Hat diese Vorgangsweise die ursprüngliche Workshopzeit verkürzt? Zeitlich gesehen ist der gesamte Workshop nicht kürzer, sondern eher länger geworden. Was wir zu den zwei Mal acht Stunden Workshop Inhalten dazu gewonnen haben, sind die Ergebnisse der Einheiten dazwischen. Dabei ging es weniger darum, noch mehr Inhalte zu bringen, sondern Angebote zu dem jeweiligen Themenbereich zu machen. Zum Beispiel wurden den Teilnehmenden extracurriculare Artikel, Podcasts, Youtube Videos, die zu dem Thema passen, zugeschickt.

Bedeutet eine Verkürzung von Einheiten online mehr Inhalt? Geht digital alles schneller? Vielleicht ist der Contentbereich komprimierter, aber dafür braucht es zu Beginn Zeit für „Icebreaker“ Aktivitäten damit sich die Menschen engagieren. Die teilnehmenden Personen können sich nicht 
ganzheitlich sehen, können sich nicht riechen, haben keinen physischen Kontakt, können sich nicht berühren, - diese Erlebnisse muss man irgendwie kompensieren und kreieren. Wir haben am Anfang viel mehr Zeit in Settings investiert, in denen sich die Teilnehmenden kennenlernen konnten. Zum Beispiel durch Breakout-Gruppen mit Erwartungsfragen zu Beginn der Veranstaltung. Wir machen das zweimal hintereinander und mischen die Gruppen dazwischen. Diese Zeit verbringen die Teilnehmenden mit ,unnessesary small talk“, wie sie es selbst betitelten.

Was wir auch gelernt haben ist, dass wir alle Multitasker geworden sind (oder so meinen wir zu behaupten). Wir sitzen alle hinter dem Rechner und nehmen nicht nur an der Videokonferenz teil, sondern sind gleichzeitig mit Mails lesen, Chat lesen, usw. beschäftigt. Zusätzlich ist in Zeiten von Home-Office Slack ${ }^{2}$ immer aktiv und jeder schaut, was so alles passiert, während man im Workshop gleichzeitig „,präsent“ ist. Multitasken - ohne dass es bemerkt wird ist jedoch ungleich schwieriger, wenn man mit einer Handvoll anderer Personen in einer Kleingruppe zusammen ist. Wenn man also eine Veranstaltung von vornherein in Kleingruppen startet, sind die Teilnehmenden auch wesentlich präsenter, weil sie zu Beginn nicht gleichzeitig woanders sind.

In der Ausnahmesituation haben sich demnach zwei Dinge grundlegend geändert. Das eine sind Zeitstrukturen und Zeitverwaltung und das andere ist eine Investition in die Selbssteuerungsfähigkeit der Teilnehmenden. Es gab noch eine dritte Erkenntnis, nämlich, dass man mehr in weniger Zeit machen konnte. Was bis dato in einem Startup wie Lyft immer schwierig war, ist, dass sich die Teilnehmenden einen oder zwei ganze Tage frei nehmen konnten oder wollten. Diese Anwesenheitsfluktuation gibt es in dem neuen Modell nicht. Es gibt diese fliegenden Wechsel nicht.

Ein anderes Riesenproblem vor COVID-19 war eine hybride Anwesenheits-Situation in den Workshops. Mitarbeitende, die nicht vor Ort im Meetingraum anwesend waren haben online an den Workshops teilgenommen. Die Variante, dass sich alle virtuell zuschalten, hat sich vor COVID-19 nie durchgesetzt. Von einem Tag auf den anderen war dieses Problem gelöst. Auf einmal waren alle virtuell und das bedeutete sehr viel Vereinfachung für die Organisator:innen der Workshops. Alle hatten nun den gleichen Zugang mit der gleichen Technik.

Was bleibt auf der Strecke, was ist der Preis, wenn man von Präsenz und/oder hybrid ganz auf virtuell umsteigt? Das Hauptargument, weshalb wir als Personalentwicklung in der Zentrale sitzen und nicht dezentral vor Ort arbeiten, ist, dass wir Leute von der gesamten Organisation funktional

\footnotetext{
2 Slack ist ein unternehmensinternes Kommunikationsprogramm.
}

zusammenbringen. Es ermöglicht einen Wissensaustausch innerhalb der Organisation und das wiederum schafft ein Netzwerk. Das war schon immer einer der Hauptgründe, warum wir als Personalentwicklungsabteilung überhaupt existieren. Wir sind eines der wenigen Systeme innerhalb der Systemlandschaft Organisation, die Leute aus verschiedensten Bereichen zusammenbringt und ihnen die Gelegenheit gibt, sich persönlich kennenzulernen und Kontakte herzustellen.

Das würde bedeuten, dass sich Mitarbeitende auch auf einer emotionalen Ebene begegnen, sodass Resonanzphänomene entstehen können. Richtig. Man baut funktionsübergreifende Beziehungen auf und auf diese eher losen Kontakte greift man zurück, sobald man in einem professionellen Kontext Personen aus den jeweiligen Bereichen braucht. Ein solcher Kontakt würde nicht stattfinden, wenn wir nicht diese Momente gehabt hätten, wo wir uns kennengelernt und diesen Wissensaustausch gehabt hätten. Im virtuellen Bereich gibt es das auf einer professionellen Ebene zwar auch jedoch nicht so sehr auf einer persönlichen Ebene. Wir versuchen das Verknüpfen dieser beiden Ebenen zu forcieren indem wir nach den Programmen die Gruppe proaktiv in Kleingruppen aufteilen, ihnen Kalendereinträge mitgeben, um sich ein, zwei oder drei Monate später für Reflexionen und Analysen zu treffen.

Ist eine empathische Kontaktaufnahme über den Bildschirm möglich? Ich glaube das ist nur bedingt möglich. Wenn jemand nervös ist, oder grundsätzlich Schwierigkeiten hat sich zu artikulieren, dann spürt man das. Im nicht-virtuellen Raum wird meistens Gelegenheit und Aufmerksamkeit geschaffen, um solche Person zu unterstützen. Virtuell findet das nur bedingt statt. Meine Erfahrung ist, unabhängig ob virtuell oder in Präsenz, dass, wenn es eine Person gibt, die den Ball ins Rollen bringt, es dann läuft. Aber es ist so viel leichter in einer Präsenzveranstaltung jemand dazu zu bringen. Die Stille in einer Anfangssituation auszuhalten fällt Menschen schwer. Diese Schweigemomente in Präsenzveranstaltungen, wo man etwas spürt habe ich virtuell noch nicht erlebt. Ganz im Gegenteil. Um Leute vom Multitasking wegzubringen muss der virtuelle Kick-off energetisch und motivierend sein. Also genau das Gegenteil von Stille.

Damit sich Leute öffnen und über Dinge reden, die ein bisschen unter die Haut gehen, muss man Intimität herstellen. Das geht in einer Präsenzveranstaltung leichter. Virtuell funktioniert das nur mittels Breakout-Gruppen. Des Weiteren war es in Präsenzveranstaltungen für Moderator:innen immer möglich, sich im Raum zu bewegen, von Gruppe zu Gruppe zu spazieren und zu beobachten. Das geht im virtuellen Raum schon auch, aber es ist unangenehm, sich von Breakout zu Breakout einzuschalten. Auf einmal ist man im Bild und kein unbemerkter Beobachter mehr der 
nur zuhört ohne zu stören. Das vermisse ich sehr. Man verliert die Überschaubarkeit der angesprochenen Themen und das Zeitbudget der Kleingruppenarbeit. Man weiß digital nicht, ob manche Gruppen schon vom Wochenende reden während andere noch am Arbeiten sind. Das vermisse ich sehr. Diese Steuerung der Gesamtgruppe, auch wenn sie in Kleingruppen arbeiten, ist extrem schwierig.

Was auch verloren gegangen ist, sind die „Water Cooler" Gespräche, bei denen sich Teilnehmende in den Pausen miteinander austauschen. „Kaffeeklatsch“ findet nicht statt.

In Präsenzveranstaltungen geht man gemeinsam in die Pause und bleibt zusammen. Im Virtuellen geht man individuell in die Pause ohne die anderen. Das ist auch etwas, das viele Teilnehmende vermissen - auf andere sprichwörtlich zugehen können, die sie interessant finden, mit denen sie gerne ins Gespräch kommen möchten. Virtuell passiert es einfach nicht, oder Moderator:innen müssen es forcieren. Es gibt kein: „Könnten wir uns mal treffen“. In Präsenz würden die Personen aufeinander zugehen, aber virtuell würde das nicht stattfinden, wenn Moderator:innen den Raum dafür nicht kreieren würden.

Was mir aufgefallen ist - die virtuelle Welt hat uns ja alle zu größeren Narzissten gemacht. Das System zeigt dir dich selbst. In Präsenzveranstaltungen zieht man nicht einen Spiegel heraus und betrachtet sich selbst. Man macht das bestenfalls auf der Toilette, nicht im Raum. Virtuell kann man zum Beispiel den eigenen Gesichtsausdruck kontrollieren, während man zuhört, spricht, oder sich anderweitig einbringt.

Um auf das Thema Zeit zurückzukommen, wie haben sich die ganzen COVID-19 bedingten Umstellungen auf die Zeitgestaltung als Manager und Führungskraft ausgewirkt? Es gibt alle möglichen Statistiken die besagen, dass Leute im Home-Office mehr arbeiten als vorher. Das physische Ineinander-Hineinlaufen im Office ist durch Multitasking am Bildschirm ersetzt worden. Gleichzeitig im Meeting, im Chat, in E-Mails zu sein. Im Meeting nur halb präsent zu sein. Das Schlimme ist, man sieht es und macht es dennoch selbst. Und es ist hierarchisch. Die jeweilige Person, die an einen berichtet, die hierarchisch niedriger ist, wird es sich nicht erlauben, Multitasking mit jemandem zu machen, der Senior ist. Der Senior hat quasi das freie Recht und die Erlaubnis, es zu tun. Unausgesprochen, nicht niedergeschrieben. Es ist skurril.

Was ich vor COVID-19 sehr sympathisch fand waren Führungskräfte, die in Meetings ihren Laptop zu machten und ihre volle Präsenz zur Verfügung stellten. Das macht momentan niemand. Denn, wenn du deinen Rechner zumachst, existierst du gar nicht mehr, d.h. der Rechner ist offen, die Programme auch. Das ist die neue Norm.

Der Gebrauch der Kommunikationsplattform Slack ist enorm angestiegen. Das In-Sich-Hineinlaufen im Office macht man mittlerweile mit Slack. Was Slack nicht ersetzt ist wiederum die Beziehungsebene. Wenn schon eine Beziehung besteht, ist es viel leichter, jemanden anzuschreiben. Daher kreieren wir auch in unseren Online Workshops diesen Zeitslot, der es ermöglicht, sich gegenseitig zu kontaktieren.

Welche Überlegungen gibt es für eine Zeit nach COVID-19? Was total spannend werden wird ist, wohin wir wieder zurück tendieren werden. Firmen in den USA arbeiten jetzt schon daran, die Mitarbeitenden wieder ins Office zurückzuholen und alles wieder in den Zustand wie vor COVID$19 \mathrm{zu}$ versetzen. Mitarbeitende selbst hingegen versuchen diesen Prozess zu stoppen und argumentieren, dass Vieles im virtuellen Raum gleich, wenn nicht sogar besser funktioniert.

Von der Weiterbildung her wollen wir zum Beispiel Onboarding komplett virtuell beibehalten. Wir wollen gar nicht, dass die Mitarbeitenden ins Office kommen, weil ja nicht jeder im gleichen Office anfängt. Das spart Zeit, Geld und schafft darüber hinaus Verteilungsgerechtigkeit. Zudem haben wir momentan die Absicht, dass, wenn es wieder zurück ins Office geht, wir den Workshop-Rhythmus von zwei Stunden pro Wochen mit intermediären Aufgaben beibehalten. Es wird Präsenzveranstaltungen geben, aber wenn wir etwas in Präsenz durchführen werden, dann müssen alle Personen anwesend sein. Ich habe auch das Gefühl, dass die Menschen jetzt ängstlich wären, acht Stunden mit anderen Leuten in einem Raum zu sitzen, oder aber Konzentrationsschwierigkeiten hätten, acht Stunden zuzuhören.

In den jetzt schon fast zwei Jahren haben wir uns daran gewöhnt, die ganze Zeit zu multitasken und uns nicht länger als eine oder zwei Stunden mit einem Thema auseinander zu setzen.

Auch wenn es wieder Präsenzveranstaltungen geben sollte, werden wir das Virtuelle beibehalten. Hybride Veranstaltungen, wie es sie vor COVID-19 gab, werden wir absichtlich vermeiden.

Wie reagieren die Organisationen auf diese Nach-COVID-19 Vorstellungen der Mitarbeitenden? Leider tendieren Organisationen nicht dahin. Sie wollen zurück zu einem Zustand wie vor COVID-19; sie wollen Mitarbeitende in Präsenz haben, weil sie annehmen, dadurch entstünde wieder ein Zusammenhalt, mehr Produktivität und Innovation.

Es wird meines Erachtens nicht funktionieren, weil, erstens sind die Menschen nicht mehr dieselben, sie sind andere. Zweitens, was bedeutet Loyalität gegenüber einer Organisation, in der man noch nie einen Fuß gesetzt hat? Und momentan argumentiert die Organisation, dass man die Kultur und das, was zwischen den Zeilen in einer Organisation steht nicht annimmt und verinnerlicht, wenn man 
nicht physisch ebendort anwesend ist. Manche Führungskräfte plädieren stark dafür, dass sie alles ganz genau so haben wollen, wie vorher. Dieses Verständnis von Produktivität gekoppelt an die physische Anwesenheit von Personen ist fest verankert. Ich habe die Hypothese, dass sich diese Personen irgendwie durch COVID-19 „durchmanagen“, um dann wieder in frühere Verhaltens- und Führungsmuster zurückkehren. Dieses Zurück geht nicht mehr. Es gibt ein neues Altes, aber kein altes Altes.

In welcher Weise wirken sich die Arbeitsbedingungen, wie beispielsweise das Arbeiten im Home-Office während der COVID-19 Zeit auf die Alltagsorganisation aus? Das ist ein Thema, das oft angesprochen wurde. Dadurch dass man Einsicht in den persönlichen Hintergrund seiner Mitarbeitenden hat, erfährt man so viel mehr über jemandes persönlichen Raum. Als Eltern in der Arbeitswelt hat das viel Empathie von Menschen, die keine Kinder haben, geschaffen. Das hat Verbindungen zu den jeweils anderen hergestellt.

Virtuelle Kommunikation und Home-Office Arbeitsplätze haben Auswirkungen auf die Leistungsbeurteilung gehabt. Die Welt ist viel diverser geworden. Ich bin oft darauf angesprochen worden, wie denn Mitarbeitende unter COVID-19 Bedingungen zu beurteilen wären. Meine Antwort war zweiseitig: in welchen Zeitfenstern konnte die Person denn überhaupt Arbeit verrichten und wie produktiv war die Person in dieser Zeit. Es ist eine enorme Verantwortung für die Manager fair zu sein und sich in die individuelle Situation der Mitarbeitenden hineinzuversetzen. Das ist extrem schwer. Unsere Verantwortung für Manager-Weiterbildung war es, einen Leitfaden zu entwickeln, wie man diese neue Situation mit den Mitarbeitenden anspricht. Das war während COVID-19 ein großer Veränderungsprozess.

Abgesehen davon sah ich eine sehr positive Entwicklung bezüglich der Leistungsevaluierung denn auf einmal waren alle sonst bestehenden Wahrnehmungsverzerrungen wie „Similar to me bias“ oder „proximity bias“ nicht mehr so stark ausgeprägt. Stattdessen wurde die effektive Produktivität in den Vordergrund gestellt, was wesentlich mehr Objektivität in der Leistungsevaluierung mit sich brachte. Meine große Sorge ist es jetzt, wie wir es schaffen werden, in einen hybriden Zustand zu kommen, indem sowohl physisch als auch nicht physisch anwesende Mitarbeitende weiterhin für deren effektive Produktivität evaluiert werden. Leider sehe ich die Zukunft diesbezüglich nicht sehr rosig. Leistungsevaluierungen werden möglicherweise noch drastischer verzerrt ausfallen als vor COVID-19. Personen, die auch in Zukunft remote arbeiten, werden sehr wahrscheinlich schlechtere Leistungsbeurteilungen bekommen, weil Vorgesetzte ihre Mitarbeitenden nicht persönlich kennengelernt haben und keine Beziehungen aufgebaut wurden (oder diese abklingen). Systemische und programmatische Gerechtigkeit diesbezüglich herzustellen, beziehungsweise diese beizubehalten, ist meine große Herausforderung für die kommenden Jahre und ich hoffe das alle, die sich mit diesem Thema auseinandersetzen, einen ähnlichen Zugang haben und Lösungsansätze etablieren werden.

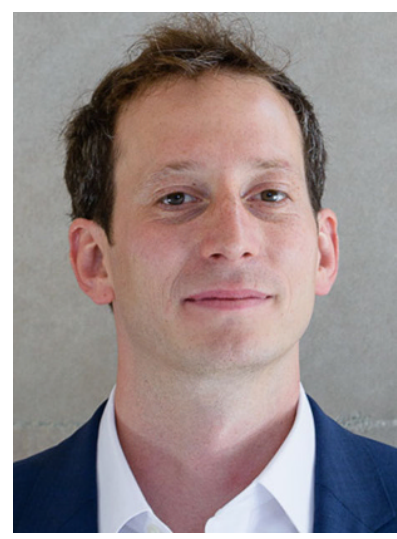

Ulrich Heitzlhofer 